\title{
Kernos
}

Revue internationale et pluridisciplinaire de religion grecque antique

16 | 2003

Varia

\section{Devenir Courète}

\section{Francesco Guizzi}

URL : http://journals.openedition.org/kernos/819

DOI : $10.4000 /$ kernos. 819

ISSN : 2034-7871

\section{Éditeur}

Centre international d'étude de la religion grecque antique

\section{Édition imprimée}

Date de publication : 1 janvier 2003

Pagination : 171-175

ISSN : 0776-3824

Référence électronique

Francesco Guizzi, « Devenir Courète », Kernos [En ligne], 16 | 2003, mis en ligne le 14 avril 2011, consulté le 02 mai 2019. URL : http://journals.openedition.org/kernos/819 ; DOI : 10.4000/kernos.819 


\section{Devenir Courète"}

Le dixième livre de la Géograpbie de Strabon traite de l'Eubée dans le premier chapitre, de l'Étolie et de l'Acharnanie, dans le deuxième, de la Crète, dans le quatrième. Le troisième chapitre est une longue digression «consacrée... aux Courètes et à leurs congénères " ${ }^{1}$, c'est-à-dire aux " collectivités mythiques " ${ }^{2}$ ou aux «Göttervereine $»^{3}$. Cette digression n'est pas placée ici au hasard. Elle « sert de transition ${ }^{4}$ entre des régions de la Grèce du nord et l'île située à l'extrémité sud de la Grèce, sous prétexte que ces quatre régions se disputaient l'origine des Courètes. La source de Strabon pour cette digression est Posidonius, mais les paragraphes 19-22 sont tirés de l'œuvre de Démétrios de Scepsis, cité sans doute déjà par Posidonius luimême . Démétrios rassemblait quelques opinions sur l'origine des Courètes qu'il voulait réfuter. Parmi celles-ci, une tradition concerne la provenance rhodienne des Courètes $^{6}$ :

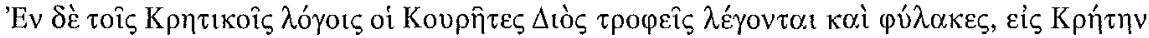

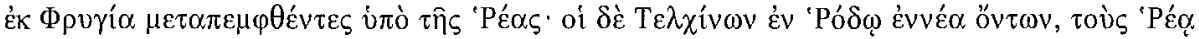

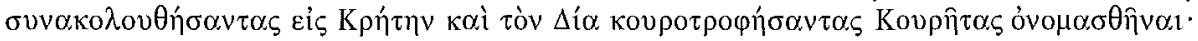

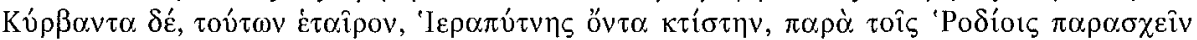

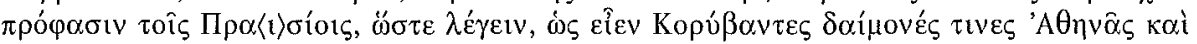
'H $\lambda$ íov $\pi \alpha \hat{i} \delta \varepsilon \varsigma$.

Dans les Traditions crétoises, les Courètes paraissent en qualité de gardiens et d'éducateurs de Zeus que Rhéa elle-même aurait fait venir de Phrygie en Crète, tandis que d'autres traditions enseignent que ceux des neuf Telchines de Rhodes qui accompagnent Rhéa en Crète pour s'occuper de Zeus, alors adolescent, portaient le nom de Courètes et que Cyrbas, l'un des leurs, le fondateur d'Hiérapytna, servit d'argument aux Praesiens devant les Rhodiens pour leur représenter que les Corybantes étaient des divinités nées de l'union d'Hélios et d'Athéna.

Cet article, qui a été présenté à Rhodes lors du VIII ${ }^{\mathrm{e}}$ Congrès du CIERGA, se fonde sur les résultats d'un travail bien plus détaillé qui est publié dans le premier chapitre de F. GuizzI, "Hierapytna. Storia di una polis cretese dalla fondazione alla conquista romana", $M A L$ ser. IX, 13 (2001), p. 275-444, spéc. p. 281-303. Je me dois de remercier mon beau-frère Bertrand Lemaire qui, en sortant du domaine de l'algèbre qu'il domine en maître, a bien voulu corriger et améliorer mon français.

1 H. Jeanmaire, Couroi et Courètes, Lille, 1939, p. 593.

2 A. Brelich, Gli eroi greci. Un problema storico-religioso, Roma, 1958, p. 325-351.

3 W. Burkert, Griecbische Religion der archaischen und klassiscben Epoche, Stuttgart, 1977 , p. $268-271$,

4 Fr. Lasserre, Strabon. Géograpbie. Livie $X$, t. VII, Paris, 1971, p. 7.

5 Cf. F. Mora, "L'interpretazione delle collettività divine in Strabone $(X, 3)$ e la fenomenologia religiosa di Posidonio", SMSR 59 (1993), p. 7-19.

6 Strabon, X, 3, 19 (C472), trad. Fr. Lasserre. Cf. Eustathe, Commentaril ad lliadem, p. 771. 
On s'aperçoit tout de suite que ces quelques lignes posent bien des problèmes. Tout d'abord, l'existence d'une tradition, de provenance inconnue, sur l'origine rhodienne des Courètes, à laquelle Démétrios opposait une autre tradition sur leur origine phrygienne, contenue dans des Kretikoi logoi. Ensuite, le caractère originel de ces personnages mythiques qui auraient été des Telchines. Cela signifie qu'ils n'étaient pas nés Courètes, mais qu'ils l'étaient devenus en protégeant et en élevant Zeus. Ensuite encore, la présence parmi eux d'un personnage nommé Kyrbas, donc d'un Corybante «hypostase » des Corybantes, fondateur de la cité crétoise d'Hiérapytna. Nous assistons donc à un syncrétisme plus large encore que celui entre Courètes et Telchines. Enfin, le passage de Strabon nous offre des indications sur la formation - ou du moins l'utilisation - de cette tradition, dans un contexte historique, politique et culturel, même s'il est très difficile, pour ne pas dire impossible, de le reconstituer exactement.

Je partirai du fondateur d'Hiérapytna, Kyrbas. Si l'on rapproche du passage de Strabon le lemme 'I $\varepsilon \rho \alpha ́ \pi v \tau v \alpha$ d'Étienne de Byzance, qui garde la mémoire des noms anciens de la ville, dont le premier est Kyrba, on en déduit que Kyrbas n'est pas seulement fondateur, mais aussi éponyme de Kyrba-Hiérapytna. Sa nature très complexe permet parfois de le rapprocher de personnages appartenant à l'un ou à l'autre des groupes de dieux. Mais elle impose parfois de souligner très nettement les différences entre lui et les autres.

On ne pourra pas l'identifier au Kyrbas qui conduit les troupes crétoises lors de l'expédition indienne de Dionysos chez Nonnos de Panopolis ${ }^{7}$ pour deux raisons. Tout d'abord, il vient de Cnossos et sous-entend une présence de Corybantes dans toute la Crète. En outre, Nonnos ne semble pas identifier Courètes et Corybantes, puisqu'il considère les premiers comme les parents ou les ä̈euls des seconds ${ }^{8}$. Le Kyrbas de Strabon devient un Courète selon une conception qui présuppose l'existence des Corybantes et qui voit dans les Courètes le produit d'une évolution fonctionnelle, c'est-à-dire des services qu'ils rendront à Rhéa après son accouchement.

Mais Kyrbas est avant tout l'un des neuf Telchines qui viennent de Rhodes pour assister Rhéa. C'est là le côté le plus complexe du syncrétisme. Non pas pour la présence de Telchines en Crète. Des Rhodiens comme le poète Simias et des Crétois avaient même soutenu la thèse de l'origine crétoise des Telchines, attestée par l'un des noms anciens de l'île, Te $\lambda \chi 1 v i \alpha^{9}$. Leur fonction de propoloi de la Mère des dieux pourrait sembler un peu plus problématique. Mais, d'après une tradition rhodienne, les Telchines avaient élevé un autre fils de Rhéa, Poséidon, tâche qu'ils pouvaient

7 NonNos, Dionysiaques XIV, 33-35.

8 Ibid. XIII, 155. - Mais déjà chez Euripide (Bacch., 120 sq.), Courètes et Corybantes sont considérés comme un seul groupe lié au culte de la Mère Rhéa. Cf. E.R. Donos, Euripide. Bacchae, Oxford, 1944, p. 80 ad loc; J.B. Lidov, "Pindar's 'Hymn to Cybele' (fr. 80 SM): Meter, Form und Syncretism", GRBS 37 (1996), p. 129-144. Sur le culte de la Mère, cf. Burkert, o.c. (n. 3), p. 276-278; E. LANE (éd.), Cybele, Attis, and Related Cults. Essays in Memory of M. Vermaseren, Leiden, 1996 (Religions in the Graeco-Roman World, 131). I.M. Linforth, "The Corybantic Rites in Plato", Univ. of Callfornia Publications in Classical Pbilology 13 (1946), p. 157, remarquait qu'à Athènes, à l'époque classique, les rites corybantiques ne semblaient pas liés au culte de la Mère des dieux.

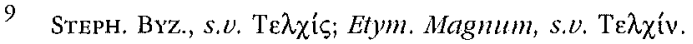


bien accomplir grâce à leur nature amphibie ${ }^{10}$. Leur fonction de paidotrophoi peut s'accorder avec la tradition rapportée par Strabon sur leur kourotrophie.

Trouver un exemple de fondation de ville par un Telchine est plus difficile. Brelich

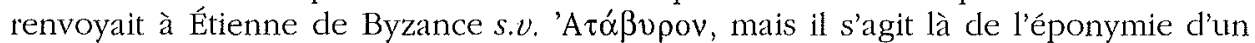
mont et non d'une polis ${ }^{11}$. Par contre, nous disposons d'un petit corpus de témoignages sur des fondations de Courètes en Crète. C'est toujours Étienne de Byzance qui livre l'information. Dans son lemme Kpń $\imath$, il cite - sans y souscrire l'hypothèse selon laquelle l'île prend son nom de Koupń $\varsigma^{12}$. Quatre poleis crétoises faisaient remonter leur origine à un Courète :

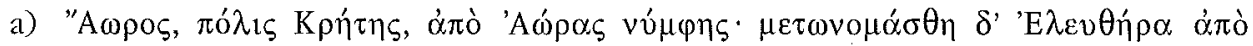

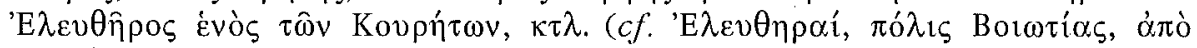

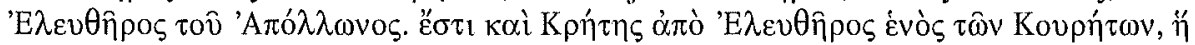

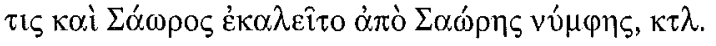

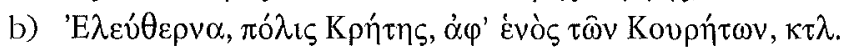

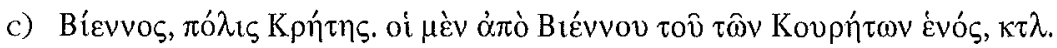

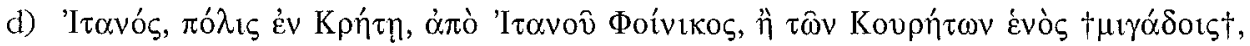
$\kappa \tau \lambda$.

On pourrait ajouter, pour que le dossier soit complet, les témoignages sur Cnossos de Jérôme et de Georges Syncelle dont la source est le Chronicon d'Eusèbe. Ces témoignages ne sont toutefois pas immédiatement comparables à ceux tirés d'Étienne de Byzance, parce que, dans le cas de Cnossos, il s'agirait d'une fondation collective des Courètes qui, par ailleurs, sont explicitement assimilés aux Corybantes, la fondation d'un sanctuaire de Cybèle leur étant aussi attribuée ${ }^{13}$. Bornons-nous donc aux témoignages d'Étienne de Byzance et remarquons que le passage de Strabon sur Kyrbas pourrait s'insérer dans ce petit corpus, malgré la complexité du personnage en question et la difficulté issue de l'assimilataion des Telchines aux Courètes.

Venons-en maintenant au dernier point de cette étude. Strabon, et sa source, font allusion à une démarche des citoyens de Praisos envers les Rhodiens. Nous disposons alors d'un repère chronologique sûr. La date de la destruction de Praisos par les Hiérapytniens intervient juste après la mort de Ptolémée VI Philométor en 145 av. J.-

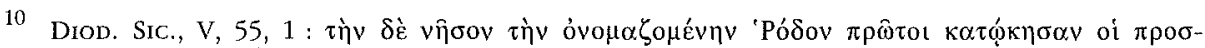

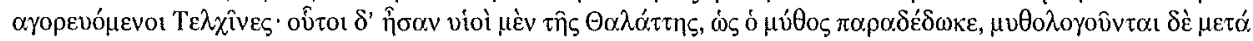

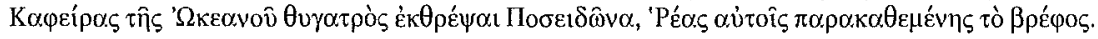

11 Brelich, o.c. (n. 2), p. 336.

12 Cf. Eustathe, ad Dionysium Periegetem, 498.

13 Jérôme, Chron., p. $22 \mathrm{~b}$ Helm : sous la $9^{\mathrm{e}}$ année du règne d'Apis à Sicyone $=53^{\mathrm{e}}$ à partir d'Abram $=1964$ av. J.-C. : Aput Cretam regnauit primus Cres indigena. Sous la $13^{\mathrm{e}}$ ou $14^{\mathrm{e}}$ année du règne d'Apis à Sicyone $=58^{\mathrm{e}}$ ou $57^{\mathrm{e}}$ à partir d'Abram $=1960$ ou 1959 av. J.-C.): Creta dicta a Crete indigena, quem aiunt unum Curetanum fuisse, a quibus Iuppiter absconditus est et nutritus. Hi Cnoson ciuitatem in Creta condiderunt et Cybelae matris templum. Cf. aussi ibid., p. 26b Helm : sous la $9^{\mathrm{e}}$ année du règne d'Aegydius à Sicyone $=130^{\mathrm{e}}$ à partir d'Abram $=1887$ av. J.-C. : In Creta regnaut primus Cres indigena, ut quidam uolunt. Alii uero supra scriptum tempus uindicant; SYNC, Ecloga Cbronograpbica, 236, 19 - 237, 4 Dindorf = 145, 19-24 Mosshammer (cf. EusèBe,

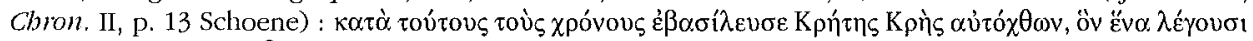

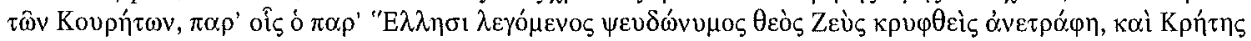

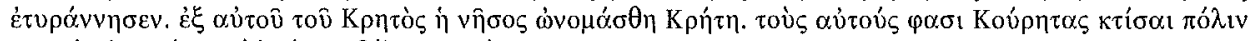

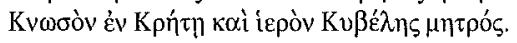


C. ${ }^{14}$ Cette date fournit un terminus ante quem pour la formation de cette tradition, ce qui s'accorde bien avec la date de l'œuvre de Démétrios de Scepsis ${ }^{15}$. Le contexte historique dans lequel les Praisiens auraient utilisé ce mythe reste obscur. Mais nous ne nous tromperons pas en plaçant cet épisode entre le début du $\mathrm{III}^{\mathrm{e}}$ siècle, qui voit Hiérapytna apparaître sur la scène crétoise en signant un traité avec Praisos ${ }^{16}$ et 145 av. J.-C, ${ }^{17}$ Nous pourrions même aller un peu plus loin et situer l'épisode entre le début de la première « guerre crétoise » que Rhodes déclencha contre les «pirates » crétois vers 205 av. J.-C. et la cleuxième guerre crétoise que se déroula entre 155 et 153 av. J.-C., c'est-à-dire durant la période où Rhodes fut plus active en Crète orientale ${ }^{18}$.

Il reste à savoir ce que voulaient obtenir les Praisiens, sur la base du mythe de fondation de Hiérapytna selon lequel les Corybantes étaient fils d'Athéna et d'Hélios, le « Hautpgot» et la « Hauptgöttin » de Rhodes ${ }^{19}$. Voulaient-ils souligner un lien entre Hiérapytna et Rhodes, par ailleurs évident dans la nature de Telchine de Kyrbas et dans la toponymie plus ancienne de la polis crétoise, à l'avantage de, ou bien contre, Hiérapytna? Je crois qu'il est impossible de répondre. Mais il faudra réfléchir encore sur deux aspects du mythe et de son interprétation par les Praisiens.

Tout d'abord, celui de la personnalité divine de cette Athéna. Rien de surprenant dans les rapports de la déesse avec Hélios à Rhodes ${ }^{20}$. Athéna est aussi en rapport avec les Telchines qui lui avaient élevé une statue en tant que Te $\lambda \chi \imath v i ́ \alpha$, c'est-à-dire $\beta$ ó, $\sigma \kappa \alpha v o \zeta$, par allusion à son regard funeste ${ }^{21}$. Quand les Praisiens parlaient d'une

14 Sur la destruction de Praisos par les Hiérapytniens, nous sommes informés par un passage de Strabon (X, 4, 12 [C478/9]) et un passage de l'arbitrage de Magnésie du Méandre entre les Hiérapytniens et les Itaniens (I.Cret. III, IV, $9=$ S.L. Ager, International Arbitrations in the Greek. World, 337-90 B.C., Berkeley, 1996, 158 II, 1. 42-47). Ce dernier offre le repère chronologique en rappelant la succession entre la mort de Ptolémée et la destruction de la ville crétoise.

15 Démétrios fut l'auteur d'une polémique contre Cratès de Mallos qui participa à la célèbre ambassade à Rome en 168 av. J.-C. Cf. par ex. F. MONTANARI, "Pergamo", in Lo spazio letterario della Grecia antica 1, 2, Rome, 1993 , p. 651-652.

16 I.Cret III, Iv, 1.

17 Sur la mort de Ptolémée VI Philométor, G. HöLb, A History of the Ptolemaic Empire, London, 2001 [or. all. Darmstadt, 1994], p. 194, et W. Huss, Agypten in bellenistischer Zeit 332-330 v. Cbr:, München, 2001, p. 588.

18 Sur la première "guerre crétoise », P. PERLMAN, "KRETES AEI LESTAI? The Marginalization of Crete in Greek Thought and the Role of Piracy in the Outbreak of the First Cretan War", in P. Gabrielsen (éd.), Hellenistic Rhodes, Politics, Culture and Society, Aahrus, 1999, p. 132-161. Sur les deux « guerres crétoises », V. Gabrielsen, The Naval Aristocracy of Hellenistic Rhodes, Aahrus, 1997 (Studies in Hellenistic Civilization, 6), p. 53-56, et A. Chaniotis, Die Verträge zwischen kietischen Poleis in der bellenistischen Zeit, Stuttgart, 1996 (HABES, 24), p. 39-40 (1 ${ }^{\text {re }}$ guerre) et 49 ( $2^{\mathrm{e}}$ guerre), avec la bibliographie récente.

19 Ainsi H. van Gelder, Geschichte der alten Rhodier, Den Haag, 1900, p. 290 (pour Hélios) et 313 (pour Athéna).

20 Le dieu avait conseillé à ses descendants, les Héliades, d'instituer le culte de la déesse aussitôt que possible pour qu'elle s'établisse à Rhodes et non à Athènes. Et c'est pour cela que les sacrifices liés à ce culte étaient sans feu (Zénon de Rhodes, dans Dıoo. Sic., V, 56, 5-7 = FGrHist 523 F 1 Jacoby). Ce n'est pas la peine d'insister sur la signification des sacrifices sans feu et sur leur caractère « primordial».

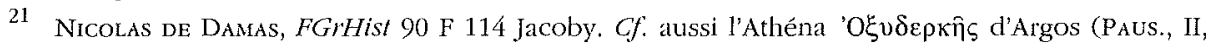

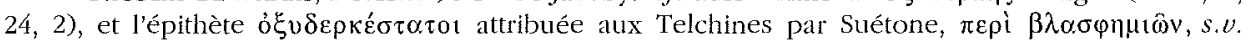

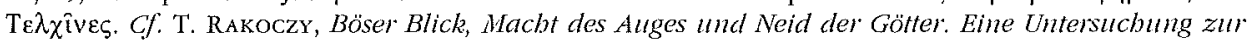
Kraft des Blickes in der griecbischen Literatur; Tübingen, 1996, p. 49, n. 442; 166-167; C. BriLlANTE, "L'invidia dei Telchini e l'origine delle arti", Aufidus 19 (1993), p. 7-42, spéc. p. 13-15. 
Athéna mère des dieux, en la considérant comme la mère des Corybantes, pensaientils à un culte particulier, peut-être ancien, de la déesse en Crète ? Certes, Athéna était vénérée en Crète à l'époque mycénienne, comme Potnia (Kn V 52.1), mais c'est aller trop vite en besogne que de rapprocher cette $A$-ta-na po-ti-ni-ja des textes mycéniens d'une déesse mère d'un groupe de divinités à la fonction de propoloi. Il faut cependant tenir compte du fait que les Praisiens étaient considérés - et se considéraient - comme les dépositaires des trađitions les plus anciennes de l'île, en tant qu'appartenant à l'etbnos étéocrétois ${ }^{22}$.

En conclusion, on devra se demander pourquoi les Praisiens soulignaient les points de contact entre Rhodes et Hiérapytna à travers ce mythe de fondation. Seraitce parce que les Hiérapytniens utilisaient le mythe de façon exactement contraire? Praisos était depuis longtemps la ville la plus puissante des communautés étéocrétoises et contrôlait le sanctuaire de Zeus Dictéen, autour duquel se réunissait un petit groupe de poleis, qui peut être considéré comme une amphictionie ${ }^{23}$. Dans le sanctuaire, on vénérait le Zeus imberbe qui serait né tout près de là, sur le mont Dikté, et qui aurait fondé la première ville de l'île en souvenir de son enfance ${ }^{24}$. Le

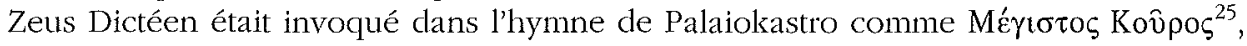
c'est-à-dire le plus grand des kouroi. Et les kouroi sont aussi les Courètes, que l'on considère la signification du mot homérique кov́ $\rho \eta \tau \varepsilon \varsigma$, ou que l'on interprète ces divinités comme des hypostases des jeunes gens qui chantaient l'hymne (en dansant des danses coutétiques? ${ }^{26}$.

Si le Telchine Kyrbas devenait un Courète, il pouvait plus aisément être admis aux rites du sanctuaire dictéen, le plus important de la Crète orientale. Et sa présence encombrante pouvait gêner quelqu'un. Mais Kyrbas restait toujours un Telchine, ce qui pouvait, en l'occurrence, être utile auprès d'une puissance très présente sur la scène crétoise aux III $^{\mathrm{e}}$ et $\mathrm{II}^{\mathrm{e}}$ av. J.-C. : Rhodes.

Francesco GuizzI

Via Chiana 35

I - 00198 RomA

e-mail : francesco.guizzi@uniroma1.it

22 Sur la conscience « ethnique » des Étéocrétois, voir J.M. Hall, Etbnic identity in Greek antiquity, Cambridge, 1997, p. 177-179.

23 ChANIOTIS, o.c. (n. 18), p. 128, cf. p. 187, n. 1134.

24 Cf. Etym. Magmum, s.v. $\Delta \mathbf{i} \kappa \tau \eta$; Dıod. SIc., V, 70, 6. Il faut noter la contradiction entre cette tradition, qui attribue à Zeus la fondation de la première polis de l'île, et tous les mythes de fondation par les Courètes dont on a parlé plus haut.

25 I.Cret. III, II, 2.

26 Voir P. Ceccarell, La pirica nell'antichità greco romana. Studi sulla danza armata, Pisa/Roma, 1998 (Filologia e Critica, 83), p. 108 sq. et passim. 